\title{
Partial observer canonical form for multi-output nonlinear forced system: a new method
}

\author{
Haotian Xu, Jingcheng Wang, Hongyuan Wang, \\ Ibrahim Brahmia and Shangwei Zhao
Key Laboratory of System Control and Information Processing, \\ Ibrahim Brahmia and Shangwei Zhao
Key Laboratory of System Control and Information Processing, \\ Department of Automation, Ministry of Education of China, \\ Shanghai Jiao Tong University, Shanghai, China
}

\begin{abstract}
Purpose - The purpose of this paper is to investigate the design method of partial observer canonical form (POCF), which is one of the important research tools for industrial plants.

Design/methodology/approach - Motivated by the two-steps method proposed in Xu et al. (2020), this paper extends this method to the case of Multi-Input Multi-Output (MIMO) nonlinear system. It decomposes the original system into two subsystems by observable decomposition theorem first and then transforms the observable subsystem into OCF. Furthermore, the necessary and sufficient conditions for the existing of POCF are proved.

Findings - The proposed method has a wide range of applications including completely observable nonlinear system, noncompletely observable nonlinear system, autonomous nonlinear system and forced nonlinear system. Besides, comparing to the existing results (Saadi et al, 2016), the method requires less verified conditions.

Originality/value - The new method concerning design POCF has better plants compatibility and less validation conditions.
\end{abstract}

Keywords Multi-output nonlinear system, Partial observer canonical form, Two-step method,

Nonlinear observable decomposition

Paper type Research paper

\section{Introduction}

The development of modern manufacturing industry guided by intelligent manufacturing is inseparable from the basic manufacturing equipment and integrated manufacturing system. For example, complex sensor networks are widely used in power grid, transportation system and industrial objects (Estrin et al., 1999; Akyildiz et al., 2002; Deng et al., 2015, 2017; Hao et al., 2012; Li and Tong, 2016); and permanent magnet synchronous motor (PMSM) technology is widely used in modern power electronics technology, microchip technology and advanced control theory (Bae et al., 2001; Altaey and Kulaksiz, 2017; Schoonhoven and Uddin, 2016). However, the control system in the industrial plants, whether it is the sensor network or the servo system represented by the PMSM, is inseparable from the accurate measurement and estimation of the state in the system. Generally speaking, the actual industrial plants, especially the major equipment used in basic manufacturing, such as Tunnel Boring Machine (TBM) (Yang et al., 2019; Li et al., 2010; Zhao et al., 2015) and PMSM, often have strong

(C) Haotian Xu, Jingcheng Wang, Hongyuan Wang, Ibrahim Brahmia and Shangwei Zhao. Published in Journal of Intelligent Manufacturing and Special Equipment. Published by Emerald Publishing Limited. This article is published under the Creative Commons Attribution (CC BY 4.0) licence. Anyone may reproduce, distribute, translate and create derivative works of this article (for both commercial and noncommercial purposes), subject to full attribution to the original publication and authors. The full terms of this licence may be seen at http://creativecommons.org/licences/by/4.0/legalcode

This work is supported by National Natural Science Foundation of China (No.61533013, 61633019), Shaanxi Provincial Key Project (2018ZDXMGY-168). 

$\underset{1,1}{\text { JIMSE }}$

nonlinear characteristics. Therefore, the study of nonlinear system state estimation has been a hot topic during the past several decades (Elbuluk and Li, 2003; Hicham et al., 2004; Gan and Wang, 2015; Liu, 2018).

The nonlinear observer is mainly concentrated in two aspects: one is about high-gain observer and another one is about observer error linearization. The latter is beginning with a group of necessary and sufficient conditions for observer canonical form (OCF) of single output system proposed by Krener and Isidori (1983). Then many scholars popularized this theory (See in Krener and Respondek (1985), Xia and Gao (1989) and Hou and Pugh (1999) for the multi-outputs system, in Lee (2017) for verifiable conditions, in Lee et al. (2015) for restricted dynamic observer error linearizability and the reference herein).

Besides, Boutat et al. (2009) give the conditions of OCF in dual version comparing to Krener and Isidori (1983). However, the conditions proposed by Krener and Isidori (1983) and Boutat et al. (2009) are too strict to be suitable for some nonlinear systems. To this end, many articles focus on relaxing their conditions by using some specific skills, such as output diffeomorphism (Boutat and Busawon, 2011; Krener and Respondek, 1985), time scaling (Respondek et al., 2004, Wang et al., 2010), auxiliary output (Back et al., 2006), dynamic compensation (Califano and Moog, 2014), virtual output (Noh et al., 2004) and approximately linear error dynamics (Lynch and Bortoff, 2001; Deutscher and Bauml, 2010; Nam, 1997).

As for the forced nonlinear system, there are also lots of achievements, such as Krener and Respondek (1985), Jo and Seo (2002) and Tami et al. (2016). They take into account the nonlinear system in the form of $\dot{x}=f(x)+g(x, u)$. In this regard, one can regard the forced system as an autonomous system $\dot{x}=f(x)$ firstly and use the method designed for autonomous system to design the required diffeomorphism, and then put the same transformation on vector field $g(x, u)$.

However, OCF requires the nonlinear system to be completely observable, which is not satisfied and does not need to be satisfied for many practical systems. To this end, some scholars proposed Partial Observer Canonical Form (POCF) for noncompletely observable system (See in Jo and Seo (2002), Tami et al. (2016), Saadi et al. (2016), Roebenack and Lynch (2006) and some reference therein). POCF is a quasi-linearization system which decomposes the system into an observable subsystem and an unobservable subsystem, and the observable subsystem takes the form of OCF. Both of OCF and POCF are widely used in industrial plant, not only in PMSM (Elbuluk and Li, 2003; Gan and Wang, 2015) but also in sensor networks (Xu and Wang, 2019; Xu and Wang, 2020, Xu et al., 2020a, c). But up to now, the POCF of multi-outputs forced system has not been documented. In this paper, a new method for calculating POCF is proposed from the perspective of observable decomposition.

The main contributions of this paper mainly consist of: (1) Motivated by the idea of $\mathrm{Xu}$ et al. (2020b), the POCF of multi-outputs forced nonlinear system $\dot{x}=f(x)+g(x, u)$ is constructed with two steps. The first is to decomposition the original system into two subsystems by observable decomposition theorem (Isidori, 1989) and the second is to transform the observable subsystem into OCF. (2) A group of necessary and sufficient conditions about whether the original nonlinear system can be transformed by these two steps, i.e. the migration result of OCF conditions will be deduced under observable decomposition. (3) The results above have a wide range of applications including completely observable system, noncompletely observable system, autonomous system and forced system. (4) Furthermore, same as our preliminary conclusion (Xu et al., 2020b), our sufficient and necessary conditions, compared to the existing results (Jo and Seo, 2002; Tami et al., 2016), only need to verify fewer conditions.

The rest of this paper is organized as follows: Section 2 gives some previous work and formulates the problem. The main result of this paper, i.e. the two steps method and the corresponding conditions are proved in Section 3. Section 4 gives an example to show the effectiveness of our main result and we provide concluding remarks in Section 5. 
At the end of the introduction, some notations of this paper should be declared. We denote the symbol $\operatorname{col}\left\{\phi_{1}, \cdots, \phi_{n}\right\}$ as a matrix $\left[\phi_{1}^{T}, \cdots, \phi_{n}^{T}\right]^{T}$, where $\phi$ represents a matrix or a map with single output. Symbol $[\cdot]_{m \times n}$ is a matrix with row $m$ and column $n$. $L_{f} h(x)$ is Lee derivative of function $h(x)$ along to vector field $f(x) \cdot\langle\cdot, \cdot\rangle$ is the inner product between two vector fields. A Lie bracket about two vector fields $f$ and $g$ is $[f, g]$. We use * in a matrix to represent the nonzero and not important entry. And we use * in the subscript of a diffeomorphism to represent the Jacobian determinant of this diffeomorphism. For example, $\Phi_{*}=\partial \Phi(x) / \partial x^{T}$.

\section{Problem formulation and preliminaries}

Taking into account a multi-output nonlinear system as

$$
\begin{aligned}
& \dot{x}=f(x)+g(x, u), \\
& y=h(x) .
\end{aligned}
$$

where $x \in R^{n}, u \in R^{m}, y \in R^{p}$ stands for state variables, control inputs and measurement output respectively, $f(x)$ and $g_{i}(x)$ are smooth vector fields with dimension $n$, and $h(x)$ is a $p$ dimension smooth vector value function which is written as $h(x)=\left(h_{1}(x), h_{2}(x), \cdots, h_{p}(x)\right)^{T}$.

Following the definition of Krener and Respondek (1985), we define a group of codistributions

$$
\begin{gathered}
\mathcal{E}_{i} \triangleq \operatorname{span}\left\{d L_{f}^{l} h_{k} \mid 0 \leq l \leq i-1,1 \leq k \leq p\right\}, i=1,2, \cdots, n, \\
\mathcal{E}_{0} \triangleq \operatorname{span}\{0\} .
\end{gathered}
$$

Then the codimension at some point $x$ of $\mathcal{E}$ is defined as $\kappa_{i}=\operatorname{dim}\left\{\mathcal{E}_{i}(x)\right\}-\operatorname{dim}\left\{\mathcal{E}_{i-1}(x)\right\}$ for $i=1, \cdots, n$. With this description, one can state the definition of the output relative degree concerning the $k$ th output of system (1), (2) in follows (Krener and Respondek, 1985):

$$
r_{k}=\operatorname{card}\left\{\kappa_{i} \geq k \mid 1 \leq i \leq n\right\}, \quad 1 \leq k \leq p,
$$

where card $\{\cdot\}$ represents the number of elements of a set. One may get $r_{1} \geq r_{2} \geq \cdots \geq r_{p} \geq 1$ by arranging the order of output appropriately. In order to design an observer for (1), (2) with linear error dynamics, we wander a diffeomorphism such that it can transform this system into observer canonical form (OCF)

$$
\begin{gathered}
\dot{\xi}=A \xi+\gamma(y)+\sum_{k=1}^{m} a_{k}(y) u_{k}, \\
y=C \xi,
\end{gathered}
$$

where $A, C$ are the block diagonal matrices denoted as $A=\operatorname{blockdiag}\left\{A_{1}, \cdots, A_{p}\right\}$, $C=\operatorname{blockdiag}\left\{C_{1} \cdots, C_{p}\right\}$, and for all $1 \leq i \leq p$, with $A_{i}$ and $C_{i}$ satisfying

$$
A_{i}=\left[\begin{array}{cc}
0_{1 \times\left(r_{i}-1\right)} & 0 \\
I_{\left(r_{i}-1\right) \times\left(r_{i}-1\right)} & 0_{\left(r_{i}-1\right) \times 1}
\end{array}\right], C_{i}=[0, \cdots, 0,1] \in R^{r_{i}} .
$$

Both Krener and Respondek (1985) and Xia and Gao (1989) proposed the sufficient and necessary conditions for transforming (1), (2) into OCF. Before introducing the OCF conditions, some definitions and notations should be introduced. They will be used 
JIMSE 1,1

\section{4}

throughout the rest of this paper. We firstly give some codistributions defined in Xia and Gao (1989) for $i=1,2, \cdots, p$,

$$
\begin{gathered}
\Delta^{\perp}=\operatorname{span}\left\{d L_{f}^{l} h_{k} \mid 1 \leq k \leq p, 0 \leq l \leq r_{k}-1\right\}, \\
\Delta_{i}^{\perp}=\operatorname{span}\left\{d L_{f}^{l} h_{k} \backslash d L_{f}^{r_{i}-1} h_{i} \mid 1 \leq k \leq p, 0 \leq l \leq r_{i}-1\right\} .
\end{gathered}
$$

Note that there exists a nonzero orthogonal distribution $\Delta$ corresponding to codistribution $\Delta^{\perp}$ when $\operatorname{dim}\left\{\Delta^{\perp}\right\}<n$. In addition, $\Delta$ and $\Delta^{\perp}$ satisfies $\langle\omega, H\rangle=0$ for arbitrary $\omega \in \Delta^{\perp}$ and $H \in \Delta$. Then some linear equations concerning $y_{i}=h_{i}(x)$ are introduced as:

$$
\begin{aligned}
& \left\langle d L_{f}^{l-1} h_{i}, \tau_{j}\right\rangle=\delta_{i, j} \cdot \delta_{l, r_{i}}, l=1, \cdots, r_{i}, \text { if } i \leq j, \\
& \left\langle d L_{f}^{l-1} h_{i}, \tau_{j}\right\rangle=\delta_{i, j} \cdot \delta_{l, r_{i}}, l=1, \cdots, r_{j}, \text { if } i>j,
\end{aligned}
$$

where $\delta_{i, j}$ is Kronecker symbol. For the sake of statement, the linear equations in the form of (10), (11) are denoted as a symbol $L e\left(f, h_{i}\right)$. Without loss of generality, we assume $\tau_{i} \in R^{n}$ be the solution of $\operatorname{Le}\left(f, h_{i}\right)$. Let $\theta_{i, 1}=\tau_{i}, i=1,2, \cdots, p$ and further let $\theta_{i, j}=\left[\theta_{i, j-1}, f\right]$, $2 \leq j \leq r_{i}$. For the unification of symbols, the basic vector fields of $\Delta$ are set as $\theta_{r+1}, \cdots, \theta_{n}$ with $r=\sum_{i=1}^{p} r_{i}$, i.e. $\Delta=\operatorname{span}\left\{\theta_{r+1}, \cdots, \theta_{n}\right\}$. Note that $\theta_{i, j} \notin \Delta$ for all $1 \leq i \leq p, 1 \leq j \leq r_{i}$ owing to linear equations (10), (11) and property $\left\langle d L_{f}^{j+1} h_{i}, \theta_{i, k}\right\rangle=\left\langle d L_{f}^{j} h_{i}, \theta_{i, k+1}\right\rangle$ (Boutat and Busawon, 2011).

It is worth to be pointed out that $\tau_{i}$ is not the unique solution of $L e\left(f, h_{i}\right)$. In fact, it includes two degrees of freedom. One is because the number of equations in (10), (11) is less than $r$. The other one is because of $r<n$.

We denote the solution space of $L e\left(f, h_{i}\right)$ at point $x$ as $\mathcal{S}_{i}^{0}(x)$ and further denote $\mathbb{S}_{i}^{0}$ as the solution distribution which is produced by letting solution space move along to manifold (See the sketch map of the relationship between distribution and tangent space in Figure 1). We can also denote the tangent space at $x$ of distribution $\Delta$ as $\Delta(x)$. Then it is obvious that there is a subspace $\mathcal{S}_{i}(x)$ of solution space satisfying $\mathcal{S}_{i}(x) \subset \Delta(x)^{\perp}$ for arbitrary $x$ and all $1 \leq i \leq p$, where $\Delta(x)^{\perp}$ represents the orthogonal complement space of $\Delta . \mathcal{S}_{i}(x)$ is called special solution space of linear equations $L e\left(f, h_{i}\right)$. One may also denote a distribution $\widetilde{\Xi}_{i}$ as a special solution distribution corresponding to $\mathcal{S}_{i}(x)$. By choosing a solution in $\mathcal{S}_{i}(x)$ and letting it move along to manifold, the vector field $\tau_{i} \in \Im_{i}$ proposed above could be obtained.

Moreover, there is $\tau_{i}+H \in \widetilde{\Xi}_{i}^{0}$ for arbitrary $H \in \Delta$. In other words, $\widetilde{\Xi}_{i} \equiv \widetilde{\Xi}_{i}^{0} \bmod \Delta$.

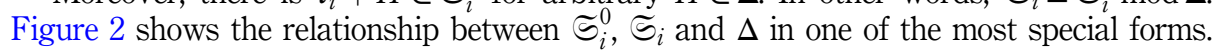

Figure 1.

Relationship between distribution and tangent subspace

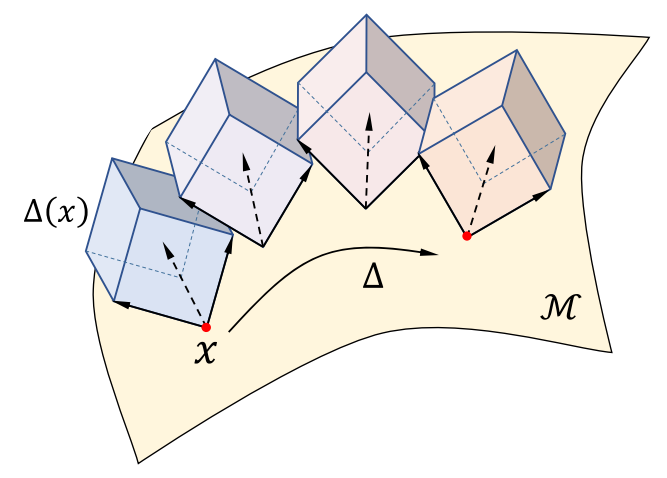




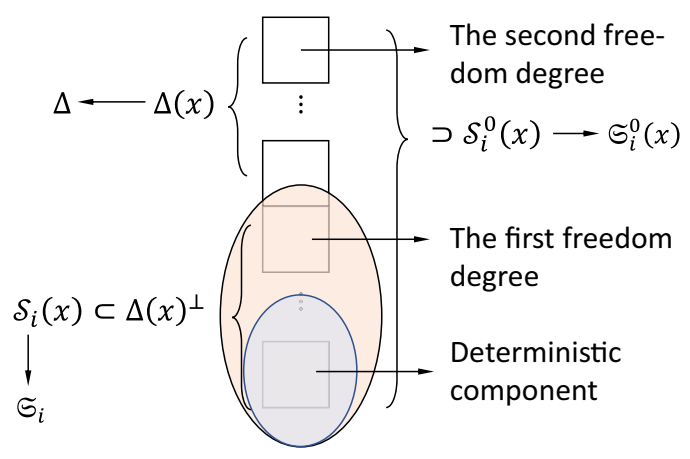

Partial observer canonical form

Figure 2.

Relationship between $\widetilde{\varsigma}_{i}^{0}, \widetilde{\varsigma}_{\mathrm{i}}$ and $\Delta$

For saving of symbol, the solution distribution and special solution distribution of $\operatorname{Le}\left(f, h_{i}\right)$ are denoted as $\widetilde{\Xi}_{i}^{0}\left(f, h_{i}\right)$ and $\widetilde{\Im}_{i}\left(f, h_{i}\right)$, respectively.

Now, one states the following Lemma.

Lemma 1. Considering a nonlinear system (1), (2) and a point in state space $x^{0}$. Then there exists a neighborhood $\mathcal{U}_{2}$ containing $x^{0}$ and a diffeomorphism $\Phi_{2}$ defined on $\mathcal{U}_{2}$ such that the underlying system can be transformed into (5), (6) if and only if

(1) The dimension of distribution $\Delta^{\perp}$ is $n$,

(2) $\operatorname{dim}\left\{\Delta_{i}^{\perp}\right\}=\operatorname{dim}\left\{\Delta^{\perp} \cap \Delta_{i}^{\perp}\right\}$,

(3) $\left[\theta_{i, l}, \theta_{j, k}\right]=0$ for all $1 \leq i, j \leq p, 1 \leq l \leq r_{i}$ and $1 \leq k \leq r_{j}$.

However, it is difficult for a multi-output system whose observable relative degree $r=\sum_{i=1}^{p} r_{i}<n$ to satisfy Lemma 1 . Thus, one hopes to find a partial observer for multioutput nonlinear which takes the form of:

$$
\begin{gathered}
\dot{\xi}_{1}=\tilde{f}_{1}\left(\xi_{1}, \xi_{2}\right)+\tilde{g}_{1}\left(\xi_{1}, \xi_{2}, u\right), \\
\dot{\xi}_{2 i}=A_{i} \xi_{2 i}+\gamma_{i}(y)+\tilde{g}_{2 i}\left(\xi_{2 i}, u\right), \\
y_{i}=C_{i} \xi_{2 i}, i=1,2, \cdots, p .
\end{gathered}
$$

where $A_{i}, C_{i}$ are in the form of (7), $\xi_{1}, \xi_{2}$ are states that transformed by POCF diffeomorphism with $\xi_{1}$ being the unobservable states and $\xi_{2}=\operatorname{col}\left\{\xi_{21}, \cdots, \xi_{2 r}\right\}$ being the observable states. So POCF (12)-(14) can be used in the situation when $\sum_{i=1}^{p} r_{i}=r<n$. As the statement of introduction, there are a lot of articles about partial observers, but only a few about them concerning the partial observers with error linearization. Moreover, to the knowledge of authors, there is few or no result about POCF of multi-output nonlinear system with input. Consequently, this paper focuses on deducing the sufficient and necessary conditions of the existence of POCF for system (1)-(2) and gives a way to calculate the corresponding diffeomorphism from the original system to POCF with a new method.

Remark 1. Conditions (1)-(3) of Lemma 1 are the sufficient and necessary conditions proposed in Xia and Gao (1989) that are used for transforming the autonomous system into OCF $\dot{z}=A z+\gamma_{1}(z)$. These conditions can also be used for forced system in which the solutions of linear equations (10), (11) are the vector fields with connection to $u$. Furthermore, there are only $i r_{i}+r_{i+1}+\cdots+r_{p}<n$ equations in linear equations (10), (11) so almost all of $\tau_{i}$, the solution of the linear equations, are not unique, except $\tau_{1}$. 


\section{JIMSE 1,1}

\section{6}

Figure 3.

Diagrammatic drawing of conditions (2) in Lemma 1
Xia and Gao (1989) treat this kind of linear equations as an improvement because it is easier for system to satisfies communicating conditions (3).

Remark 2. Conditions (2) in Lemma 1 proposed in (Xia and Gao, 1989) take the place of the requirement of Krener and Respondek (1985) that the system must be in special observable form. See the structure of $\Delta^{\perp}$ and $\Delta_{i}^{\perp}$ in Figure 3 . The red box is the codistribution $\Delta^{\perp}$, the blue box represents the vector fields spanning $\Delta_{i}^{\perp}$. Blue area is the basic vector fields of $\Delta^{\perp}$ belonging to $\Delta_{i}^{\perp}$, we denote this area as $\Delta_{b}^{\perp}$. And the yellow area named $\Delta_{s}^{\perp}$ represents the vector fields of $\Delta_{i}^{\perp}$ but not the basic vector fields in $\Delta^{\perp}$. Noting that $\Delta_{i}^{\perp}$ is contained in $\Delta^{\perp}$ because of the restriction of $\operatorname{dim}\left\{\Delta^{\perp}\right\}$. Therefore, conditions (2) actually require that all of the vector fields in $\Delta_{s}^{\perp}$ can be represented by the basic vector fields in $\Delta_{b}^{\perp}$.

\section{Main result}

At the beginning of this section, we first introduce the basic idea of the new method concerning POCF. Firstly, calculate a diffeomorphism $\Phi_{1}$ defined on a neighborhood $\mathcal{U}_{1}$ of $x^{0}$ such that the system transformed by $\Phi_{1}$ can be divided into two subsystems including observable subsystem and unobservable subsystem. Then one can design $\Phi_{2}$ by using condition in Lemma 1 to transform the observable subsystem into OCF. We thus conclude $\Phi=\Phi_{2} \circ \Phi_{1}$. The main result of this paper is to deduce the migration results of OCF conditions under observable decomposition. Noticing that the observability decomposition theorem proposed by Isidori (1989) can be easily generalized to general nonlinear systems. Hence, we have the following lemma.

Lemma 2. A distribution $\mathcal{Q}$ is called a maximum invariant distribution if it is contained in $\operatorname{span}\left\{d h_{1}, \cdots, d h_{p}\right\}^{\perp}$ and invariant under $f$ and $g$. Suppose a point $x^{0}$ and its neighborhood $\mathcal{U}_{1}$ and further suppose $\mathcal{Q}$ is involutive and nonsingular on $\mathcal{U}_{1}$. Then there is a diffeomorphism $z=\left[z_{1}^{T}, z_{2}^{T}\right]^{T}=\Phi_{1}(x)$ defined on $\mathcal{U}_{1}$ such that the system under new coordinate is

$$
\begin{gathered}
\dot{z}_{1}=\bar{f}_{1}\left(z_{1}, z_{2}\right)+\bar{g}_{1}\left(z_{1}, z_{2}, u\right), \\
\dot{z}_{2}=\bar{f}_{2}\left(z_{2}\right)+\bar{g}_{2}\left(z_{2}, u\right), \\
y_{i}=\bar{h}_{i}\left(z_{2}\right), 1 \leq i \leq p .
\end{gathered}
$$

where $\bar{f}$ and $\bar{g}$ are vector fields satisfying $\bar{f}=\operatorname{col}\left\{\bar{f}_{1}, \bar{f}_{2}\right\}=\left.\Phi_{1 *} f\right|_{x=\Phi_{1}^{-1}(z)}, \bar{g}=\operatorname{col}\left\{\bar{g}_{1}, \bar{g}_{2}\right\}=$ $\left.\Phi_{1 * g}\right|_{x=\Phi_{1}^{-1}(z)}$, and $\bar{h}(z)=\left.h(x)\right|_{x=\Phi_{1}^{-1}(z)}$.

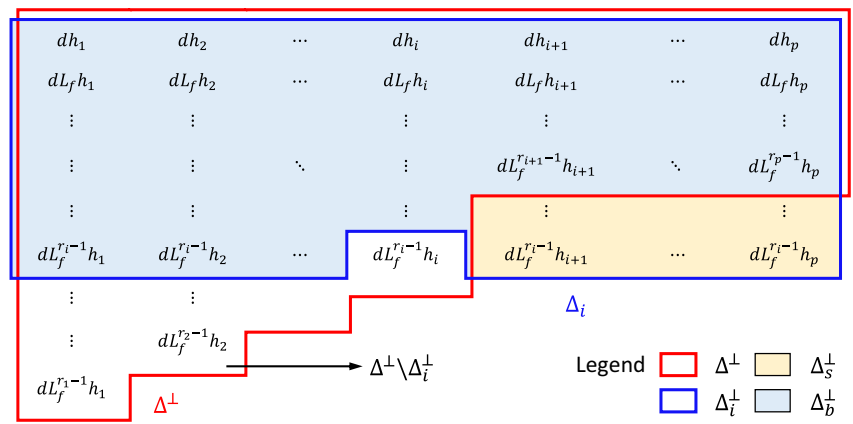


Proof. According to Frobenius theorem and Lemma in (Li, 2014, Lemma 7.1), we can obtain a diffeomorphism $z=\Phi_{1}(x)$ by the proof process of Frobenius theorem if nonsingular involutive distribution $\mathcal{Q}$ is invariant under $f$. Then $f$ can be transformed into an upper triangular form by $\Phi_{1}(x)$. Furthermore, the first $n-r$ terms of covector field $d h$ in the new coordinate will be zero. Thus, we get the observable decomposition form (15)-(17).

Partial observer canonical form

Next, some basic properties of codistribution $\Delta^{\perp}$ will be given. And some of their proof is omitted because they are direct generalizations of (Tami et al., 2016) 's Lemma.

Lemma 3. Considering a nonlinear system (1), (2). Then its codistribution $\Delta^{\perp}$ and orthogonal distribution $\Delta$ satisfies

(1) $\Delta$ and $\Delta^{\perp}$ are involutive;

(2) $\Delta$ and $\Delta^{\perp}$ are invariant under vector fields $f$;

(3) Distribution $\Delta$ is invariant under $\theta_{i, j}$ for arbitrary $1 \leq i \leq p, 1 \leq j \leq r_{i}$.

Lemma 4. For any $1 \leq i, j \leq p$, if $\tau_{i} \in \Im_{i}^{0}, \tau_{j} \in \Im_{i}^{0}$, then

(1) For all $1 \leq i \leq p$ and $\theta_{i, k}, 1 \leq k \leq r_{i}$ corresponding to $\tau_{i}$, there exists a vector field $\vartheta_{i, k}$ with $\vartheta_{i, 1} \in \Im_{i}$ such that $\theta_{i, k} \equiv \vartheta_{i, k} \bmod \Delta$;

(2) There is $\left[\theta_{i, k}+H_{1}, \theta_{j, l}+H_{2}\right] \equiv\left[\theta_{i, k}, \theta_{j, l}\right] \bmod \Delta$ for arbitrary $H_{1}, H_{2} \in \Delta$. In specially, there exists vector fields $\vartheta_{i, k}, \vartheta_{j, l}$ satisfying $\left[\theta_{i, k}, \theta_{j, l}\right] \equiv\left[\vartheta_{i, k}, \vartheta_{j, l}\right] \bmod \Delta$ for all $\theta_{i, k}, \theta_{j, l}$, where $\vartheta_{i, 1}, \vartheta_{j, 1} \in \widetilde{\Xi}_{i}$.

Lemma 5. (Li (2014) Lemma 10.1). Suppose $\phi(x)$ is a real value function. We further suppose $X, Y$ are vector fields defined on open set $U \subset \mathbb{R}^{n}$. Then for arbitrary positive integers $s, k, l$, we have

$$
\left\langle d L_{X}^{s} \phi(x), a d_{X}^{k+l} Y(x)\right\rangle=\sum_{i=1}^{l}(-1)^{i} C_{l}^{i} L_{X}^{l-i}\left\langle d L_{X}^{s+i} \phi(x), a d_{X}^{k} Y(x)\right\rangle .
$$

Furthermore, one can conclude the following two equations are equivalent:

$$
\begin{aligned}
& \text { (i) } L_{Y} \phi(x)=L_{Y} L_{X} \phi(x)=\cdots=L_{Y} L_{X}^{k} \phi(x)=0, \quad \forall x \in U, \\
& \text { (ii) } L_{Y} \phi(x)=L_{a d_{X} Y} \phi(x)=\cdots=L_{a d_{X}^{k} Y} \phi(x)=0, \quad \forall x \in U .
\end{aligned}
$$

Lemma 6. Suppose two nonlinear system $\dot{x}=f(x, u), y=h(x) \in \mathbb{R}^{p}$ and $\dot{z}=\bar{f}(z, u)$, $y=\bar{h}(z) \in \mathbb{R}^{p}$. Additionally suppose there is a neighborhood $V$ around a point $x^{0}$ and a diffeomorphism $\Pi: x \rightarrow z$ defined on $V$ such that $\bar{f}=\left.\Pi_{*} f\right|_{x=\Pi^{-1}(z)}$. Then vector field $\tau_{i}$ satisfies $\tau_{i} \in \Im_{i}\left(f, h_{i}\right)$ for all $i=1,2, \cdots, p$ if and only if $\bar{\tau}_{i}=\Pi * \tau_{i} \in \widetilde{\Xi}_{i}\left(\bar{f}, \bar{h}_{i}\right)$.

Proof. Might as well let $\bar{\theta}_{i, j}=\left.\Pi_{*} \theta_{i, j}\right|_{x=\Pi^{-1}(z)}=\left.\frac{\partial \Pi}{\partial x^{T}} \theta_{i, j}\right|_{x=\Pi^{-1}(z)}$. Then multiply this formula by $\frac{\partial \bar{h}_{i}(z)}{\partial z^{T}}$ on its both sides and we thus have,

$$
L_{\bar{\theta}_{i, j}} \bar{h}_{i}(z)=\frac{\partial h_{i}(\Pi(x))}{\partial x^{T}} \theta_{i, j}=\left.L_{\theta_{i, j}} h_{i}(x)\right|_{x=\Pi^{-1}(z)} .
$$


JIMSE 1,1

According to Lemma 5, the left half side (LHS) of the above formula is equivalent to the LHS of linear equations $\operatorname{Le}\left(\bar{f}, \bar{h}_{i}\right)$. And the right half side (RHS) of the above formula is equal to LHS of $L e\left(f, h_{i}\right)$. Therefore, $\tau_{i} \in \Im^{0}\left(f, h_{i}\right)$ if and only if $\bar{\tau}_{i}=\Pi * \tau_{i} \in \Im_{i}^{0}\left(\bar{f}, \bar{h}_{i}\right)$.

Theorem 1. $\mathcal{Q}^{\perp}=\Delta^{\perp}$ if and only if $\left[\theta_{i}, g_{k}\right] \in \Delta$ for $r+1 \leq i \leq n$ and $1 \leq k \leq m$.

Proof. $(\Rightarrow) \mathcal{Q}^{\perp}=\Delta^{\perp}$ yields $\mathcal{Q}^{\perp}=\operatorname{span}\left\{\omega_{1}, \cdots, \omega_{n}\right\}$. Since the observable relative degree is $r$, one has $\mathcal{Q}^{\perp}=\operatorname{span}\left\{\omega_{1}, \cdots, \omega_{r}\right\}(\mathrm{Li}, 2014)$, which indicates $\mathcal{Q}^{\perp}$ is invariant under $f$. Furthermore, according to the definition of $\mathcal{Q}^{\perp}, \mathcal{Q}^{\perp}$ is also invariant under $g_{k}, k=1, \cdots, m$. Then Lemma 1 yields $\mathcal{Q}=\Delta$ is invariant under $g_{k}, k=1, \cdots, m$. Bearing in mind that $\theta_{r+1}, \cdots, \theta_{n}$ are the basic vector fields of $\Delta$, one thus has $\left[\theta_{i}, g_{k}\right] \in \Delta$ for $r+1 \leq i \leq n$ and $1 \leq k \leq m$.

$(\Leftarrow)$ The proof of sufficiency can be completed by inverse deducing the above steps.

Theorem 2. Considering a nonlinear system (1), (2), and assume $\mathcal{U}$ is a neighborhood around a point $x^{0}$ in state space. If a distribution $\Delta$. corresponding to this system is nonsingular in $\mathcal{U}$ with dimension $n-r$. Then there exists a diffeomorphism $\Phi=\Phi_{2} \circ \Phi_{1}$ defined on $\mathcal{U}$ such that the original system (1), (2) can be transformed into POCF (12)-(14) if and only if

(1) $\operatorname{dim}\left\{\Delta^{\perp}\right\}=r$ and $\mathcal{Q}^{\perp}=\Delta^{\perp}$,

(2) $\operatorname{dim}\left\{\Delta_{i}^{\perp}\right\}=\operatorname{dim}\left\{\Delta^{\perp} \cap \Delta_{i}^{\perp}\right\}$,

(3) $\left[\theta_{i, k}, \theta_{j, l}\right] \in \Delta$ are fulfilled for all $\tau_{i} \in \mathbb{S}_{i}^{0}\left(f, h_{i}\right), 1 \leq i \leq p$ and arbitrary $1 \leq i, j \leq p$, $1 \leq k \leq r_{i}, 1 \leq l \leq r_{j}$

(4) $\left[\theta_{i, k}, g\right] \in \Delta, 1 \leq k \leq r_{i}-1$ for arbitrary $1 \leq i \leq p$.

Proof. Since $\Delta$ is nonsingular on the neighborhood $\mathcal{U}$ around $x^{0}$, there exists a coordinate transformation $\Phi_{1}$ such that the original system can be transformed into (15), (16). Then this proof will be finished in the following five steps.

(1) Given the following two groups of codistribution for all $i=1,2, \cdots, p$,

$$
\begin{gathered}
\bar{\Delta}^{\perp}=\operatorname{span}\left\{d L_{\bar{f}}^{l} \bar{h}_{k} \mid 1 \leq k \leq p, 0 \leq l \leq r_{k}-1\right\}, \\
\bar{\Delta}_{i}^{\perp}=\operatorname{span}\left\{d L_{\bar{f}}^{l} \bar{h}_{k} L_{\bar{f}}^{r_{i}-1} \bar{h}_{i} \mid 1 \leq k \leq p, 0 \leq l \leq r_{i}-1\right\}, \\
\overline{\mathcal{D}}^{\perp}=\operatorname{span}\left\{d L_{\bar{f}_{2}}^{l} \bar{h}_{k} \mid 1 \leq k \leq p, 0 \leq l \leq r_{k}-1\right\}, \\
\overline{\mathcal{D}}_{i}^{\perp}=\operatorname{span}\left\{d L_{\bar{f}_{2}}^{l} \bar{h}_{k} \backslash d L_{\bar{f}_{2}}^{r_{i}-1} \bar{h}_{i} \mid 1 \leq k \leq p, 0 \leq l \leq r_{i}-1\right\} .
\end{gathered}
$$

Moreover, divide the corresponding regions of the above codistributions according to the definition in Figure 3. It is supposed to prove that condition $\operatorname{dim}\left\{\Delta_{i}^{\perp}\right\}=\operatorname{dim}\left\{\Delta^{\perp} \cap \Delta_{i}^{\perp}\right\}$ holds if and only if $\operatorname{dim}\left\{\overline{\mathcal{D}}_{i}^{\perp}\right\}=\operatorname{dim}\left\{\overline{\mathcal{D}}^{\perp} \cap \overline{\mathcal{D}}_{i}^{\perp}\right\}$.

$(\Rightarrow)$ It is known that for any $k>r_{j}-1, j>i$, the covector field $d L_{f}^{k} h_{j} \in \Delta_{s}^{\perp}$ has no connection with the covector field in $\Delta^{\perp} \backslash \Delta_{i}^{\perp}$. Since the diffeomorphism does not change the independence of the vector fields and the covector fields, we conclude that the following covector fields $d L_{\bar{f}}^{k} \bar{h}_{j} \in \bar{\Delta}_{s}, k>r_{j}-1, j>i$ have no connection with covector fields in $\bar{\Delta}^{\perp} \backslash \bar{\Delta}_{i}^{\perp}$. 
Next, we will prove that $L_{\bar{f}}^{k} \bar{h}_{j}=L_{f_{2}}^{k} \bar{h}_{j}$ for arbitrary $1 \leq k \leq r_{i}-1$ and all of them are only related to $z_{2}$. This assertion will be proved by mathematical induction. Set $k=1$, it is obviously that $\bar{h}_{j}$ are only related to $z_{2}$ and hence we have $\partial \bar{h}_{j} / \partial z_{1}^{T}=0$. It follows with

$$
L_{\bar{f}} \bar{h}_{j}=\left(\begin{array}{ll}
\frac{\partial \bar{h}_{j}}{\partial z_{1}^{T}} & \frac{\partial \bar{h}_{j}}{\partial z_{2}^{T}}
\end{array}\right)\left(\begin{array}{l}
\bar{f}_{1} \\
\bar{f}_{2}
\end{array}\right)=L_{\bar{f}_{1}} \bar{h}_{j}+L_{\bar{f}_{2}} \bar{h}_{j}=L_{\bar{f}_{2}} \bar{h}_{j} .
$$

Partial observer canonical form

Noticing that both $\bar{h}_{j}$ and $\bar{f}_{2}$ are only related to $z_{2}$, so $L_{\bar{f}_{2}} \bar{h}_{j}$ is also only related to $z_{2}$. Assume this assertion is fulfilled for all $k \leq r_{i}-2$, then set $k=r_{i}-1$ and one can deduce that

$$
L_{\bar{f}}^{r_{i}-1} \bar{h}_{j}=L_{\bar{f}} L_{\bar{f}}^{r_{i}-2} \bar{h}_{j}=L_{\bar{f}} L_{\bar{f}_{2}}^{r_{i}-2} \bar{h}_{j}=\left(\frac{\partial L_{\bar{f}_{2}}^{r_{i}-2} \bar{h}_{j}}{\partial z_{1}^{T}} \frac{\partial L_{\bar{f}_{2}}^{r_{i}-2} \bar{h}_{j}}{\partial z_{2}^{T}}\right)\left(\begin{array}{l}
\bar{f}_{1} \\
\bar{f}_{2}
\end{array}\right)=L_{\bar{f}_{2}}^{r_{i}-1} \bar{h}_{j} .
$$

It is apparent to show that $L_{\bar{f}_{2}}^{r_{i}-1} \bar{h}_{j}$ is only related to $z_{2}$ owing to $\partial L_{\bar{f}_{2}}^{r_{i}-2} \bar{h}_{j} / \partial z_{1}^{T}=0$. Therefore, we obtain $d L_{\bar{f}}^{k} \bar{h}_{j}=\left(0, d L_{\bar{f}_{2}}^{k} \bar{h}_{j}\right)$ for all $r_{j} \leq k \leq r_{i}-1$, where $d L_{\bar{f}_{2}}^{k} \bar{h}_{j} \in \overline{\mathcal{D}}_{s}^{\perp}$.

Let $N_{i}=r-\left(i r_{i}+r_{i+1}+\cdots+r_{p}-1\right)$ and denote $\bar{\Delta}^{\perp} \backslash \bar{\Delta}_{i}^{\perp}$ as $\operatorname{span}\left\{\bar{\omega}_{1}, \cdots, \bar{\omega}_{N_{i}}\right\}$. Since $\bar{\omega}_{l}$ can be represented by $\bar{\omega}_{l}=\left(0, \bar{v}_{l}\right)$ for $\forall l=1,2, \cdots, N_{i}$, we have $\overline{\mathcal{D}}^{\perp} \backslash \overline{\mathcal{D}}_{i}^{\perp}=$ span $\left\{\bar{v}_{1}, \cdots, \bar{v}_{N_{i}}\right\}$. Thereby, it can be deduced for arbitrary family of smooth functions $c_{1}(z), \cdots, c_{N_{i}}(x)$ that

$$
d L_{\bar{f}}^{k} \bar{h}_{j}=\left(0, d L_{\bar{f}_{2}}^{k} \bar{h}_{j}\right)=\sum_{l=1}^{N_{i}} c_{l}(z) \bar{\omega}_{l}=\sum_{i=1}^{N}\left(0, c_{l}(z) \bar{v}_{l}\right)=0,
$$

if and only if $c_{1}(z)=\cdots=c_{N_{i}}(z)=0$. Hence, all the covector fields $d L_{\bar{f}_{2}}^{k} \bar{h}_{j} \in \overline{\mathcal{D}}_{s}^{\perp}$, $k>r_{i}-1, j>i$ have no connection with the covector fields of $\overline{\mathcal{D}}^{\perp} \backslash \overline{\mathcal{D}}_{i}^{\perp}$.

$(\Leftarrow)$ The proof of sufficiency can be completed by inverse deducing the above steps.

(2) It can be directly deduced by the definition of observable relative degree that $\operatorname{dim}\left\{\Delta^{\perp}\right\}=\operatorname{dim}\left\{\overline{\mathcal{D}}^{\perp}\right\}=r$.

(3) Considering linear equations $\operatorname{Le}\left(\bar{f}, \bar{h}_{i}\right)$ and $\operatorname{Le}\left(\bar{f}_{2}, \bar{h}_{i}\right)$, where $1 \leq i \leq p$. Assume $\bar{\tau}_{i} \in \widetilde{\Im}_{i}^{0}\left(\bar{f}, \bar{h}_{i}\right), \widetilde{\tau}_{\mathrm{i}} \in \widetilde{\Im}_{i}^{0}\left(\bar{f}_{2}, \bar{h}_{i}\right)$ and let $\bar{\theta}_{i, 1}=\bar{\tau}_{i}, \bar{\theta}_{i, k}=\left[\bar{\theta}_{i, k-1}, \bar{f}\right], k=2, \cdots, r_{i}$ and $\tilde{\theta}_{i, 1}=\tilde{\tau}_{i}, \tilde{\theta}_{i, k}=\left[\tilde{\theta}_{i, k-1}, \bar{f}_{2}\right], k=2, \cdots, r_{i}$. What need to be proved in this step is that there exists a family of proper solutions $\vartheta_{i, 1} \in \widetilde{S}_{i}\left(f, h_{i}\right)$ and $\theta_{i, 1} \equiv \vartheta_{i, 1} \bmod \Delta$ such that $\left[\theta_{i, k}, \theta_{j, l}\right] \in \Delta$ for all $1 \leq i, j \leq p$ and $1 \leq k \leq r_{i}, 1 \leq l \leq r_{j}$ if and only if $\left[\tilde{\theta}_{i, k}, \tilde{\theta}_{j, l}\right]=0$.

By comparing linear equations $\operatorname{Le}\left(\bar{f}, \bar{h}_{i}\right)$ and $\operatorname{Le}\left(\bar{f}_{2}, \bar{h}_{i}\right)$, we know $\left(0, \tilde{\tau}_{i}^{T}\right)^{T}=\bar{\tau}_{i}$ $=\bar{\vartheta}_{i, 1} \in \Im_{i}\left(\bar{f}, \bar{h}_{i}\right)$. Therefore, it can be directly checked that $\bar{\vartheta}_{i, k}=\left[\bar{\vartheta}_{i, k-1}, f\right]=\left(0, \tilde{\theta}_{i, k}^{T}\right)^{T}$ for arbitrary $\bar{\vartheta}_{i, k}, 2 \leq k \leq r_{i}$. Sequentially,

$$
\left[\bar{\vartheta}_{i, k}, \bar{\vartheta}_{j, l}\right]=\left[\left[\begin{array}{c}
0 \\
\tilde{\theta}_{i, k}
\end{array}\right],\left[\begin{array}{c}
0 \\
\tilde{\theta}_{j, l}
\end{array}\right]\right]=\left[\begin{array}{c}
0 \\
\left.\tilde{\theta}_{i, k}, \tilde{\theta}_{j, l}\right]
\end{array}\right] .
$$

As a result, $\left[\bar{\vartheta}_{i, k}, \bar{\vartheta}_{j, l}\right]=0$ if and only if $\left[\tilde{\theta}_{i, k}, \tilde{\theta}_{j, l}\right]=0$. According to Lemma 4, we can obtain $\left[\bar{\theta}_{i, k}, \bar{\theta}_{j, l}\right] \equiv\left[\bar{\vartheta}_{i, k}, \bar{\vartheta}_{j, l}\right] \bmod \bar{\Delta}$ by choosing $\bar{\theta}_{i, k} \equiv \bar{\vartheta}_{i, k} \bmod \bar{\Delta}$ and $\bar{\theta}_{j, l} \equiv \bar{\vartheta}_{j, l} \bmod \bar{\Delta}$. Then we get $\left[\bar{\theta}_{i, k}, \bar{\theta}_{j, l}\right] \in \bar{\Delta}$ are equivalent to $\left[\tilde{\theta}_{i, k}, \tilde{\theta}_{j, l}\right]=0$. According to Lemma 6 , we know $\bar{\tau}_{i}=\Phi_{1 *} \tau_{i}$, which infers $\bar{\theta}_{i, k}=\Phi_{1 *} \theta_{i, k}$. We can thus deduce that 
JIMSE

1,1

$$
\left[\theta_{i, k}, \theta_{j, l}\right]=\Phi_{1 *}^{-1}\left[\bar{\theta}_{i, k}, \bar{\theta}_{j, l}\right] \in \Delta \Leftrightarrow\left[\tilde{\theta}_{i, k}, \tilde{\theta}_{j, l}\right]=0 .
$$

(4) Prove $\left[\theta_{i, j}, g\right] \in \Delta$ is equivalent to $\left[\tilde{\theta}_{i, j}, \bar{g}_{2}\right]=0$ for arbitrary $1 \leq i \leq p, 1 \leq j \leq r_{i}-1$.

It is known according to Lemma 3 that $\Delta$ is invariant under $f, g$. Bearing in mind $\bar{\theta}_{i, j} \equiv \bar{\vartheta}_{i, j}$ mod $\Delta$ (Lemma 4$\}$ ), we have

$$
\begin{gathered}
\Phi_{1^{*}}\left[\theta_{i, j}, g\right]=\left[\bar{\theta}_{i, j}, \bar{g}\right] \equiv\left[\bar{\vartheta}_{i, j}, \bar{g}\right] \bmod \bar{\Delta} \\
\equiv\left[\left[\begin{array}{c}
0 \\
\tilde{\theta}_{i, j}
\end{array}\right],\left[\begin{array}{c}
\bar{g}_{1} \\
\bar{g}_{2}
\end{array}\right]\right] \bmod \bar{\Delta} \equiv\left[\begin{array}{c}
0 \\
\left.\tilde{\theta}_{i, j}, \bar{g}_{2}\right]
\end{array}\right] \bmod \bar{\Delta} .
\end{gathered}
$$

Therefore, $\left[\theta_{i, j}, g\right] \in \Delta$ if and only if $\left[\tilde{\theta}_{i, j}, \bar{g}_{2}\right]=0$.

(5) Prove conditions (1)-(3) are necessary and sufficient.

$(\Rightarrow)$ Since there is coordinate transformation $z=\Phi_{1}(x)$ defined on $\mathcal{U}_{1}$ such that the original system can be transformed into (12), (13). If there is a diffeomorphism $\xi=\Phi(x)$ defined on $\mathcal{U}$ such that the original system can be transformed into (5), (6). Then there must exist a $\xi=\Phi_{2}(z)$ on $V_{2}=\Phi_{1}\left(\mathcal{U}_{2}\right)$ such that $\Phi$ is a diffeomorphism defined on $\mathcal{U}=\mathcal{U}_{1} \cap \mathcal{U}_{2}$ and satisfies $\Phi=\Phi_{2} \circ \Phi_{1}$. Noticing that $\Phi_{2}$ can transform the observable subsystem into OCF (6), thus we know from Lemma 1 that $\operatorname{dim}\left\{\overline{\mathcal{D}}^{\perp}\right\}=r, \operatorname{dim}\left\{\overline{\mathcal{D}}_{i}^{\perp}\right\}=\operatorname{dim}\left\{\overline{\mathcal{D}}^{\perp} \cap \overline{\mathcal{D}}_{i}^{\perp}\right\}$ and $\left[\tilde{\theta}_{i, k}, \tilde{\theta}_{j, l}\right]=0$, which indicate conditions (1)-(3) are fulfilled due to the proof steps (1)-(3).

$(\Leftarrow)$ Because conditions (1)-(3) are fulfilled, by using Lemma 1 and proof steps (1)-(3), we deduce that there is a neighborhood $V_{2}$ around $z^{0}=\Phi_{1}\left(x^{0}\right)$ and a coordinate transformation $\Phi_{22}$ such that the observable subsystem (13) can be transformed into OCF (6). Let $\Phi_{21}=\mathscr{I}_{n-r}, \mathcal{U}_{2}=\Phi_{1}^{-1}\left(V_{2}\right)$ and construct $\Phi_{2}=\operatorname{col}\left\{\mathscr{I}_{n-r}, \Phi_{22}\right\}$. Hence we have a diffeomorphism $\Phi=\Phi_{2} \circ \Phi_{1}$ defined on $\mathcal{U}=\mathcal{U}_{1} \cap \mathcal{U}_{2}$ which can transform the original system into POCF (12), (13).

It is noted that Theorem 1 is very similar to Lemma 1 since a distribution can be spanned by a vector field 0 . Thus, we can deduce a corollary from Theorem 1 which can be used for almost all smooth affine nonlinear system with observable relative degree $0<r \leq n$. Moreover, this conclusion can be used to design POCF whether it is a single output system or a multi-output system.

Corollary 1. Consider a nonlinear system (1), (2). Suppose $\mathcal{U}$ is a neighborhood around arbitrary point $x^{0}$ in state space. If codistribution $\Delta^{\perp}$ is nonsingular on $\mathcal{U}$, then there is a diffeomorphism $\xi=\Phi(x)$ defined on $\mathcal{U}$ such that system (12)-(14) can be transformed into (5), (6) if and only if

(1) $\operatorname{dim}\left\{\Delta_{i}^{\perp}\right\}=\operatorname{dim}\left\{\Delta^{\perp} \cap \Delta_{i}^{\perp}\right\}$,

(2) $\left[\theta_{i, k}, \theta_{j, l}\right] \in \Delta$ are satisfied for all $\tau_{i} \in \widetilde{S}_{i}^{0}\left(f, h_{i}\right), 1 \leq i \leq p$ and arbitrary $1 \leq i, j \leq p$, $1 \leq k \leq r_{i}, 1 \leq l \leq r_{j}$.

There is no doubt that it is amount to Theorem 1 when $r<n$. In addition, if $r=n$, then $\operatorname{dim}\left\{\Delta^{\perp}\right\}=n$. It indicates $\Delta=\operatorname{span}\{0\}$. So, conditions (2) in Corollary 1 is equivalent to $\left[\theta_{i, k}, \theta_{j, l}\right]=0$. Thus, this corollary degenerates into Lemma 1 when $r=n$. In the situation of $p=1$, i.e. system (1), (2) is a single-output system. This corollary can deal with the same problem as what Tami et al. (2016) does. But the conditions in this conclusion are weaker than that in (Tami et al.,2016). If the system is autonomous, then this corollary degenerates into the problem discussed in Saadi et al. (2016). However, one may notice that the conditions in Saadi et al. (2016) are redundant comparing to Corollary 1. 


\section{Example}

In this section, an example will be used to demonstrate the effect of Theorem 1 and Corollary 1. Consider the following double outputs nonlinear system

$$
\left\{\begin{array}{l}
\dot{x}_{1}=-x_{3}^{2}+x_{1}^{3} x_{3}-1 / 2 x_{3}^{3}+x_{2}^{5}-5 x_{4}^{5}+x_{3} x_{1} u^{2}, \\
\dot{x}_{2}=x_{1}-1 / 2 x_{3}^{2} \\
\dot{x}_{3}=-x_{3}+x_{1}^{3}-1 / 2 x_{3}^{2}+x_{1} u^{2} \\
\dot{x}_{4}=-2 x_{4}-1 / 2 x_{2}^{2}+x_{2} \sin u \\
\dot{x}_{5}=-x_{5}-x_{1}^{2}+x_{4}^{2}-1 / 2 x_{3}^{2} \\
y_{1}=h_{1}(x)=x_{2}, y_{2}=h_{2}(x)=x_{4} .
\end{array}\right.
$$

It can be checked by definition of observable relative degree that $r_{1}=2$ and $r_{2}=1$. One can calculate directly that $\Delta^{\perp}$ equals $\mathcal{E}_{2}$. The basic covector fields of $\Delta^{\perp}$ is $[0,1,0,0,0]$, $\left[1,0,-x_{3}, 0,0\right]$ and $[0,0,0,1,0]$. Thus, the basic vector field of $\Delta$ is $\theta_{4}=\left[x_{3}, 0,1,0,0\right]^{T}$ and $\theta_{5}=[0,0,0,0,1]^{T}$. One may notice that $g(x, u)$ have no effect on the observable relative degree in this example. Because $\Delta_{1}=\operatorname{span}\left\{d h_{1}, d h_{2}\right\}$, conditions (1) in Corollary 1 are fulfilled. After solving linear equations (10), (11), one can obtain

$$
\begin{gathered}
\theta_{1,1}=[1,0,0,0,0]^{T} \in \Im_{1}, \\
\theta_{1,2}=\left[3 x_{1}^{2} x_{3}+x_{3} u^{2}, 1,3 x_{1}^{2}+u^{2}, 0,-2 x_{1}\right]^{T}, \\
\theta_{2,1}=[0,0,0,1,0]^{T} \in \Im_{2} .
\end{gathered}
$$

Note that $\theta_{1,2}=3 x_{1}^{2} \theta_{4}-2 x_{1} \theta_{5}$, so $\theta_{1,2} \in \Delta$ and then there exists $\vartheta_{1,2}=[0,1,0,0,0]^{T}$, and then it is easy to check $\left[\vartheta_{1,1}, \vartheta_{1,2}\right]=0,\left[\vartheta_{1,1}, \vartheta_{2,1}\right]=0$ and $\left[\vartheta_{1,2}, \vartheta_{2,1}\right]=0$. Therefore, system (30) satisfies all of the two conditions in Corollary 1 . Now we calculate $\Phi_{1}$ based on observable decomposition. Solve the following partial differential equation

$$
\frac{\partial \Phi_{1}}{\partial x^{T}}=\Lambda \triangleq\left[\begin{array}{ccccc}
0 & 0 & 0 & 0 & 1 \\
0 & 0 & 1 & 0 & 0 \\
0 & 1 & 0 & 0 & 0 \\
1 & 0 & -x_{3} & 0 & 0 \\
0 & 0 & 0 & 1 & 0
\end{array}\right]
$$

and get $z_{1}=\phi_{1}(x)=x_{5}, z_{2}=\phi_{2}(x)=x_{3}, z_{3}=\phi_{3}(x)=x_{2}, z_{4}=\phi_{4}(x)=x_{1}-\frac{1}{2} x_{3}^{2}$ and $z_{5}=\phi_{5}(x)=x_{4}$, where the first two rows of matrix $\Lambda$ are arbitrary two covector fields which are independent to $\Delta^{\perp}$. Therefore system (30) can be transformed by $\Phi_{1}$ into

$$
\left\{\begin{array}{l}
\dot{z}_{1}=-\left(z_{4}+1 / 2 z_{2}^{2}\right)^{2}-1 / 2 z_{2}^{2}-z_{1}+z_{5}^{2}, \\
\dot{z}_{2}=\left(z_{4}+1 / 2 z_{2}^{2}\right)^{3}-1 / 2 z_{2}^{2}-z_{2}+z_{2} u+\left(z_{4}-1 / 2 z_{2}^{2}\right) u^{2} \\
\dot{z}_{3}=z_{4}, \\
\dot{z}_{4}=z_{3}^{5}-5 z_{5}^{5}, \\
\dot{z}_{5}=1 / 2 z_{3}^{2}-2 z_{5}+z_{3} u \sin u, \\
y_{1}=z_{3}, y_{2}=z_{5} .
\end{array}\right.
$$

$\Phi_{2}$ of this example is obvious. Set $\xi_{1}=z_{1}, \xi_{2}=z_{2}, \xi_{3}=z_{4}, \xi_{4}=z_{3}$ and $\xi_{5}=z_{5}$ and we have POCF as following 


\section{JIMSE 1,1}

$$
\left\{\begin{array}{l}
\dot{\xi}_{1}=-\left(\xi_{3}+\frac{1}{2} \xi_{2}^{2}\right)^{2}-\frac{1}{2^{2}} \xi_{2}^{2}-\xi_{1}+\xi_{5}^{2}, \\
\dot{\xi}_{2}=\left(\xi_{3}+\frac{1}{2^{2}}\right)^{2}-\frac{1}{2} \xi_{2}^{2}-\xi_{2}+\xi_{2} u+\left(\xi_{3}-\frac{1}{2} \xi_{2}^{2}\right) u^{2}, \\
\dot{\xi}_{3}=\xi_{4}^{5}-5 \xi_{5}^{5}, \\
\dot{\xi}_{4}=\xi_{3}, \\
\dot{\xi}_{5}=\frac{1}{2} \xi_{4}^{2}-2 \xi_{5}+\xi_{4} u \sin u, \\
y_{1}=\xi_{4}, y_{2}=\xi_{5} .
\end{array}\right.
$$

\section{Conclusion}

To investigate the design method of POCF for a class of MIMO nonlinear system, this paper, motivated by the two steps methods of Single-output nonlinear system (Xu et al., 2020b), has generated the corresponding new methods for MIMO system. In this regard, the underlying system is divided into observable subsystem and unobservable subsystem first and then the former is transformed as OCF. Furthermore, a corollary at the end of this paper has been developed as a uniform theorem for the existing of POCF for a large class of nonlinear system, such as single-output system, multi-output system, observable system and noncompletely observable system that is considered in this paper and so on.

\section{References}

Akyildiz, I.F., Su, W., Sankarasubramaniam, Y. and Cayirci, E. (2002), “A survey on sensor networks”, IEEE Communications Magazine, Vol. 40 No. 8, pp. 102-114.

Altaey, A. and Kulaksiz, A.A. (2017), "Stability analysis of sensorless speed control of ipmsm", IEEJ Transactions on Electrical and Electronic Engineering, Vol. 17 No. 2, pp. 101-112.

Back, J., Yu, K.T. and Jin, H.S. (2006), "Dynamic observer error linearization", Automatica, Vol. 42 No. 12 , pp. 2195-2200.

Bae, B.H., Sul, S.K., Kwon, J.H. and Shin, J.S. (2001), "Implementation of sensorless vector control for super-high speedpmsm of turbocompressor", IEEE Transactions on Industrial Applications, Vol. 39 No. 3, pp. 1203-1209.

Boutat, D. and Busawon, K. (2011), "On the transformation of nonlinear dynamical systems into the extended nonlinear observable canonical form”, International Journal of Control, Vol. 84 No. 1, pp. 94-106.

Boutat, D., Benali, A., Hammouri, H. and Busawon, K. (2009), "New algorithm for observer error linearization with a diffeomorphism on the outputs", Automatica, Vol. 45 No. 10, pp. 2187-2193.

Califano, C. and Moog, C. (2014), "The observer error linearization problem via dynamic compensation", IEEE Transactions on Automatic Control, Vol. 59 No. 9, pp. 2502-2508.

Deng, F., Guo, S., Zhou, R. and Chen, J. (2015), "Sensor multifault diagnosis with improved support vector machines", IEEE Transactions on Automation Science and Engineering, Vol. 14 No. 2, pp. 1053-1063.

Deng, F., Guan, S., Yue, X., Gu, X., Chen, J., Lv, J. and Li, J. (2017), "Energy-based sound source localization with low power consumption in wireless sensor networks", IEEE Transactions on Industrial Electronics, Vol. 64 No. 6, pp. 4894-4902. 
Deutscher and Bauml (2010), "Design of nonlinear observers with approximately linear error dynamics using multivariable legendre polynomials", International Journal of Robust and Nonlinear Control, Vol. 16 No. 15, pp. 709-727.

Elbuluk, M. and Li, C. (2003), "Sliding mode observer for wide-speed sensorless control of pmsm drives", IEEE Industry Applications Conference, Ohio, pp. 480-485.

Estrin, D., Govindan, R., Heidemann, J. and Kumar, S. (1999), "Next century challenges: scalable coordination in sensor networks", 'Proceedings of the 5th Annual ACM/IEEE International Conference on Mobile Computing and Networking, pp. 263-270.

Gan, M. and Wang, C. (2015), "An adaptive nonlinear extended state observer for the sensorless speed control of a pmsm”, Mathematical Problems in Engineering, Vol. 1, pp. 1-14.

Hao, Yang, Bin, Jiang, Huaguang and Zhang (2012), "Stabilization of nonminimum phase switched nonlinear systems with application to multiagent system", Systems and Control Letters, Vol. 61 No. 10, pp. 1023-1031.

Hicham, F., Mohamed, D., Abdellatif, R. and Pierre, B. (2004), "Sliding mode observer for position and speed estimations in brushless dc motor (bldcm)", 'IEEE International Conference on Industrial Technology, Hammanmet, pp. 121-126.

Hou, M. and Pugh, A.C. (1999), "Observer with linear error dynamics for nonlinear multi-output systems”, Systems and Control Letters, Vol. 37 No. 1, pp. 1-9.

Isidori, A. (1989), Nonlinear Control Systems, Springer-Verlag Berlin Heidelberg, New York.

Jo, N. and Seo, J. (2002), "Observer design for non-linear systems that are not uniformly observable", International Journal of Control, Vol. 75 No. 5, pp. 369-380.

Krener, A.J. and Isidori, A. (1983), "Linearization by output injection and nonlinear observers", Systems and Control Letters, Vol. 3 No. 1, pp. 47-52.

Krener, A.J. and Respondek, W. (1985), "Nonlinear observers with linearizable error dynamics", SIAM Journal on Control and Optimization, Vol. 23 No. 2, pp. 197-216.

Lee, H.G. (2017), "Verifiable conditions for multi-output observer error linearizability", IEEE Transactions on Automatic Control, Vol. 62 No. 9, pp. 4876-4883.

Lee, H.G., Kim, K.D. and Jeon, H.T. (2015), "Restricted dynamic observer error linearizability", Automatica, Vol. 53 No. 11, pp. 171-178.

Li, D. (2014), Theoretical Basis of Nonlinear Control Systems, Tsinghua University Press, Beijing.

Li, Y. and Tong, S. (2016), "Adaptive neural networks decentralized ftc design for nonstrict-feedback nonlinear interconnected large-scale systems against actuator faults", IEEE Transactions on Neural Networks and Learning Systems, Vol. 28 No. 11, pp. 2541-2554.

Li, X., Yu, H., Yuan, M., Wang, J. and Yin, Y. (2010), "Dynamic modeling and analysis of shield tbm cutterhead driving system", Journal of Dynamic Systems, Measurement, and Control, Vol. 132 No. 4, pp. 1-14.

Liu, B. (2018), "Speed control for permanent magnet synchronous motor based on an improved extended state observer", Advances in Mechanical Engineering, Vol. 10 No. 1, pp. 157-162.

Lynch, A.F. and Bortoff, S.A. (2001), "Nonlinear observers with approximately linear error dynamics: the multivariable case", Automatic Control IEEE Transactions on, Vol. 45 No. 6, pp. 927-932.

Nam, K. (1997), "An approximate nonlinear observer with polynomial coordinate transformation maps", Automatic Control IEEE Transactions on, Vol. 42 No. 4, pp. 522-527.

Noh, D., Jo, N.H. and Seo, J.H. (2004), "Nonlinear observer design by dynamic observer error linearization", Automatic Control IEEE Transactions on, Vol. 49 No. 10, pp. 1746-1753.

Respondek, W., Pogromsky, A. and Nijmeijer, H. (2004), "Time scaling for observer design with linearizable error dynamics”, Automatica, Vol. 40 No. 2, pp. 277-285.

Roebenack, K. and Lynch, A.F. (2006), "Observer design using a partial nonlinear observer canonical form", International Journal of Applied Mathematics and Computer Science, Vol. 16 No. 3, pp. 333-343. 

$\underset{1,1}{\text { JIMSE }}$
Saadi, W., Boutat, D., Zheng, G. and Sbita, L. (2016), "Multi-output partial nonlinear observer normal form", IEEE Conference on Decision and Control, pp. 7654-7658.

Schoonhoven, G. and Uddin, M.N. (2016), "Mtpa and fw based robust nonlinear speed control of ipmsm drive using lyapunov stability criterion”, IEEE Transactions on Industry Applications, Vol. 52 No. 2, pp. 4365-4347.

Tami, R., Zheng, G., Boutat, D., Aubry, D. and Wang, H. (2016), "Partial observer normal form for nonlinear system", Automatica, Vol. 64 No. C, pp. 54-62.

Wang, Y., Lynch and Alan, F. (2010), "Multiple time scalings of a multi-output observer form", IEEE Transactions on Automatic Control, Vol. 55 No. 4, pp. 966-971.

Xia, X.H. and Gao, W.B. (1989), "Nonlinear observer design by observer error linearization”, SIAM Journal on Control and Optimization, Vol. 27 No. 1, pp. 199-216.

$\mathrm{Xu}, \mathrm{H}$. and Wang, J. (2019), "Distributed observer design for omniscience asymptotically aimed at a class of nonlinear system", IEEE Conference on Decision and Control, pp. 3303-3308.

$\mathrm{Xu}, \mathrm{H}$. and Wang, J. (2020), "Distributed observer-based control law with better dynamic performance based on distributed high-gain observer", International Journal of Systems Science, Vol. 51 No. 4, pp. 631-642.

Xu, H., Wang, J., Wang, B. and Wang, H. (2020a), “An improved distributed nonlinear observer for leader-following consensus via differential geometry approach", arXiv2002.00365, pp. 1-14

$\mathrm{Xu}, \mathrm{H}$., Wang, J., Wang, H. and Zhao, S. (2020b), "Partial observer canonical form design method for siso affine nonlinear system with simple validation conditions", International Journal of Control, Automation, and Systems, pp. 1-8.

Xu, H., Wang, J., Wang, H., Zhao, S. and Lin, H. (2020c), "Distributed observer design for achieving omniscience asymptotically over time-variant disconnected communication networks", 2020 IFAC World Congress, pp. 1-6.

Yang, X., Zhang, L., Xie, W. and Zhang, J. (2019), "Sequential and iterative distributed model predictive control of multi-motor driving cutterhead system for tbm", IEEE Access, Vol. 7, pp. 46977-46989.

Zhao, Y., Wang, J., Zhang, L. and Tao, H. (2015), "Fuzzy-pid based induction motor control and its application to tbm cutter head systems", International Conference on Intelligent Robotics and Applications, pp. 511-522.

\section{Corresponding author}

Jingcheng Wang can be contacted at: jcwang@sjtu.edu.cn

For instructions on how to order reprints of this article, please visit our website:

www.emeraldgrouppublishing.com/licensing/reprints.htm

Or contact us for further details: permissions@emeraldinsight.com 